\title{
Chagas' Disease and HIV Co-infection: Genotypic Characterization of the Trypanosoma cruzi Strain
}

\section{Raquel S Pacheco/ ${ }^{+}$, Marcelo S Ferreira*, Maria Inês Machado*, Célia MM

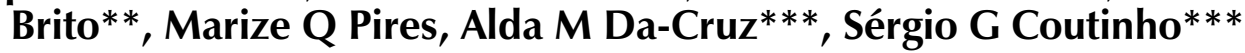

\begin{abstract}
Departamento de Bioquímica e Biologia Molecular, Instituto Oswaldo Cruz, Av. Brasil 4365, 21045-900 Rio de Janeiro, RJ, Brasil *Departamento de Patologia, Universidade Federal de Uberlândia, Uberlândia, MG, Brasil **Departamento de Ciências Biológicas, Escola Nacional de Saude Pública, Fiocruz, Rio de Janeiro, RJ, Brasil ****Departamento de Protozoologia, Instituto Oswaldo Cruz, Rio de Janeiro, RJ, Brasil
\end{abstract}

In the past few years, new aspects of the immunopathology of Chagas' disease have been described in immunosuppressed patients, such as fatal central nervous system lesions related to the reactivation of the parasite. This article is the first description of the genotypic characterization, at the strain level, of Trypanosoma cruzi isolated from a patient with Chagas" disease/AIDS co-infection. The presence of four hypodense lesions was observed in the cranial compute tomographic scan. The diagnosis of AIDS was assessed by the detection of anti-HIV antibodies using enzyme-linked immunosorbent assay (ELISA) and Western blot techniques. The CD4+ lymphocyte counts were maintained under $200 \mathrm{cells} / \mathrm{mm}^{3} \mathrm{dur}$ ing one year demonstrating the severity of the state of immunosuppression. Chagas' disease was confirmed by serological and parasitological methods. Trypomastigote forms were visualized in a thick blood smear. The parasite isolated is genotypically similar to the CL strain. The paper reinforces that cerebral Chagas' disease can be considered as another potential opportunistic infection in AIDS resulting from the reactivation of a dormant $\mathrm{T}$. cruzi infection acquired years earlier.

Key words: Chagas' disease/AIDS co-infection - opportunistic infection - Trypanosoma cruzi - reactivation

Recent reports in the literature have provided evidence for the persistence and reactivation of protozoan parasites in humans despite the treatment and apparent cure of the disease (Saravia et al. 1990, Guevara et al. 1993, Aebischer 1994). In addition reactivation of some protozoosis in patients infected by the human immunodeficiency virus (HIV) indicates that the phenomenum of parasite persistence may be the rule instead of the exception (Alvar 1994).

Chagas' disease, an infection caused by Trypanosoma cruzi, is widely distributed in the majority of the countries of the American continent from the south of the United States to the south of Argentina (PAHO 1990). In Brazil, according to Dias (1989) it is estimated that six milion people are infected by T. cruzi. A great number of chagasic patients are now migrating from rural zones to the large cities where the possibilities of acquiring the HIV are greater. In the past few years, new aspects of the immunopathology of Chagas' disease have

This work received financial support from the International Atomic Energy Agency (IAEA, Vienna, Austria). ${ }^{+}$Corresponding author. Fax: +55-21-590.3495

Received 25 August 1997

Accepted 8 January 1998 been described in immunosuppressed patients, some of them showing fatal central nervous system (CNS) lesions related to the reactivation of the parasite (Del Castillo et al. 1990, Ferreira et al. 1991, Gluckstein et al. 1992, Rocha et al. 1994, Ferreira 1996).

The present paper describes the isolation and the genotypic characterization of a $T$. cruzi strain from a Brazilian patient with Chagas' disease/ AIDS co-infection in which four cerebral lesions were found.

\section{CASE REPORT}

A 27-year-old chronic chagasic bisexual male with a history of intravenous drug use and resident in Uberlândia, Minas Gerais was admitted to the Hospital of the Federal University of Uberlândia complaining of weight loss, dry cough and fever. The physical examination revealed enlargement of cervical lymph nodes and liver. Electrocardiographic alterations were also found. Laboratory studies showed a white blood cell count of 2,700/ $\mathrm{mm}^{3}$ with $4 \%$ band forms and $30 \%$ lymphocytes. Later, his overall clinical condition worsened presenting a neutrophile count of $1,000 / \mathrm{mm}^{3}$ without band forms, $17 \%$ lymphocytes and a platelet count of $27,000 / \mathrm{mm}^{3}$. In cultures of pulmonary secretion Klebsiella pneumonie was isolated and subse- 
quently Pseudomonas alcaligenes, Staphylococcus aureus and Acinetobacter calcoaceticus. A cranial computed tomographic scan demonstrated the presence of four hypodense lesions. Serology was negative for Toxoplasma gondii but positive for $T$. cruzi. The patient died of intracranial bleeding but neither histopathologic examination nor the preparation of brain specimen cultures were carried out due to the non-authorization of his family.

\section{LABORATORY STUDIES}

Serologic and parasitological tests - The diagnosis of AIDS was assessed by the detection of anti-HIV antibodies using enzyme-linked immunosorbent assay (ELISA) and Western blot techniques. The diagnosis of Chagas' disease was confirmed by serological and parasitological methods. Indirect immunofluorescence and ELISA techniques were performed. The parasitological investigation was performed three times over a three-month period and consisted of thick blood smears for demonstration of the parasite (Fig. 1), hemoculture in LIT medium, xenodiagnosis with 40 nymphs of Triatoma infestans and blood innoculation in mice for isolation. The xenodiagnosis was shown to be the best test for the diagnosis of the parasitemia. In the first examination carried out in 1993, 100\% of the nymphs were positive. Two months later, $70 \%$ were found positive.

Follow-up examinations of the immunological condition of the patient were carried out at different time points during one year. The T-cell phenotypes were analyzed by flow cytometry (Coulter, EPICS $751^{\hat{a}}$ ) using the following monoclonal antibodies: T3-RD1, T4-FITC and T8-RD1 (Coulter). The $\mathrm{CD} 4^{+}$lymphocyte counts remained under 200 cells $/ \mathrm{mm}^{3}$ during the study demonstrating the severity of the state of immunesuppression (Fig. 2). An examination of the cellular immune response

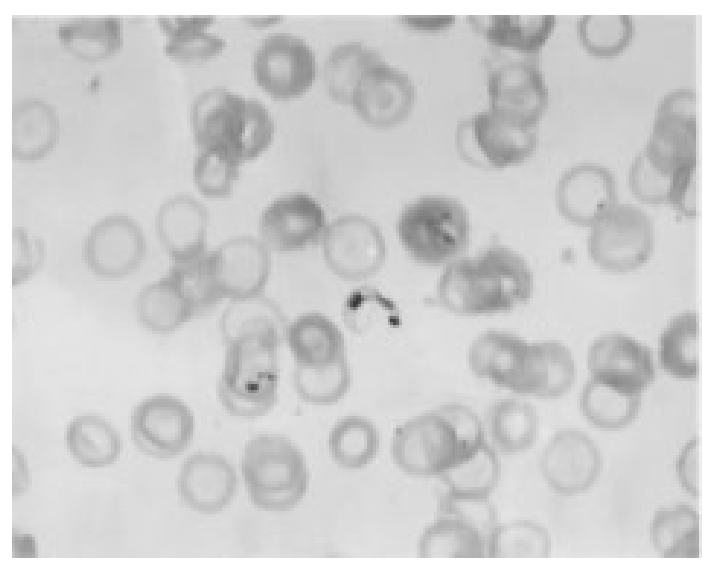

Fig. 1: trypomastigote forms found in the patient's blood ( $\mathrm{x}$ $1,000)$.

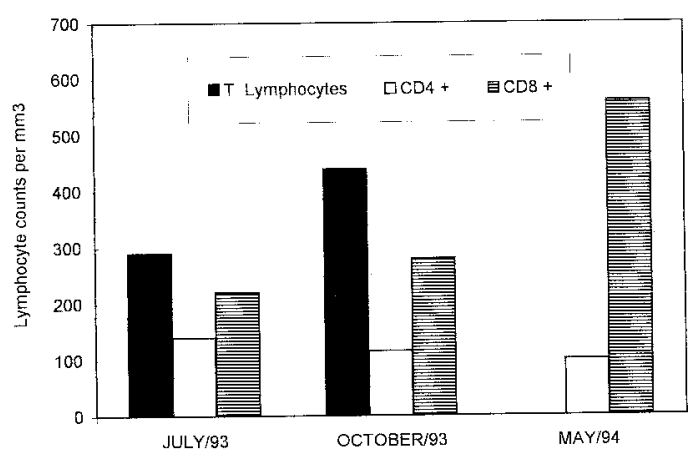

Fig. 2: T-lymphocyte subsets in the peripheral blood mononuclear cells of the patient.

was also carried out by determining the lymphocyte proliferative response of peripheral blood mononuclear cells (PBMC) induced in vitro by $T$. cruzi antigens. Briefly, PBMC were separated using a Ficoll-Hypaque (Histopaque 1077, Sigma) gradient. The PBMC ( $3 \times 10^{5}$ per well) were cultured in triplicate in round-bottomed 96-well microtitre plates for five days at $37^{\circ} \mathrm{C}$ in a humidified atmosphere of $5 \%$ of $\mathrm{CO}_{2}$, in the presence of $4 \mathrm{mg}$ of concanavalin A per well and $10^{6}$ sonicated epimastigotes of T. cruzi . Sixteen hours before harvesting, $1 \mathrm{mCi}[3 \mathrm{H}]$ thymidine (Amersham) was added to each well and the radioactivity uptake was measured in a scintillation b counter (Packard 1800). Cell proliferation is considered positive when the stimulation index is 32.5 . The lymphoproliferative index of the T-cell microcultures was very low $(<2.5)$ showing that $\mathrm{T}$ lymphocytes were not able to respond properly to antigens or mitogen.

Genotypic characterization of the parasite Epimastigotes from culture were submitted to kDNA extraction and digestion with restriction enzyme according to the protocols described by Morel et al. (1980) and Pacheco et al. (1986). Conditions for radiolabelling and hybridization have also been previously published (Pacheco et al. 1994). By using a synthetic oligonucleotide specific for the subgenus Schizotrypanum of Trypanosoma (Pacheco et al. 1996) and total kDNAs in Southern and dot blot hybridizations it was possible to characterize the human isolate as T.(S) cruzi. The kDNA restriction profile obtained with the enzyme Eco RI demonstrated a genotypic pattern similar, although not identical to the CL strain of T. cruzi. Furthermore, Southern blot hybridization of minicircle fragments revealed a high copy number of homologous sequences when kDNA of the CL strain was used as probe (Fig. 3). No sequence homology was detected between the kDNA from the parasite and a kDNA probe from the Colombian strain of T. cruzi (not shown). 


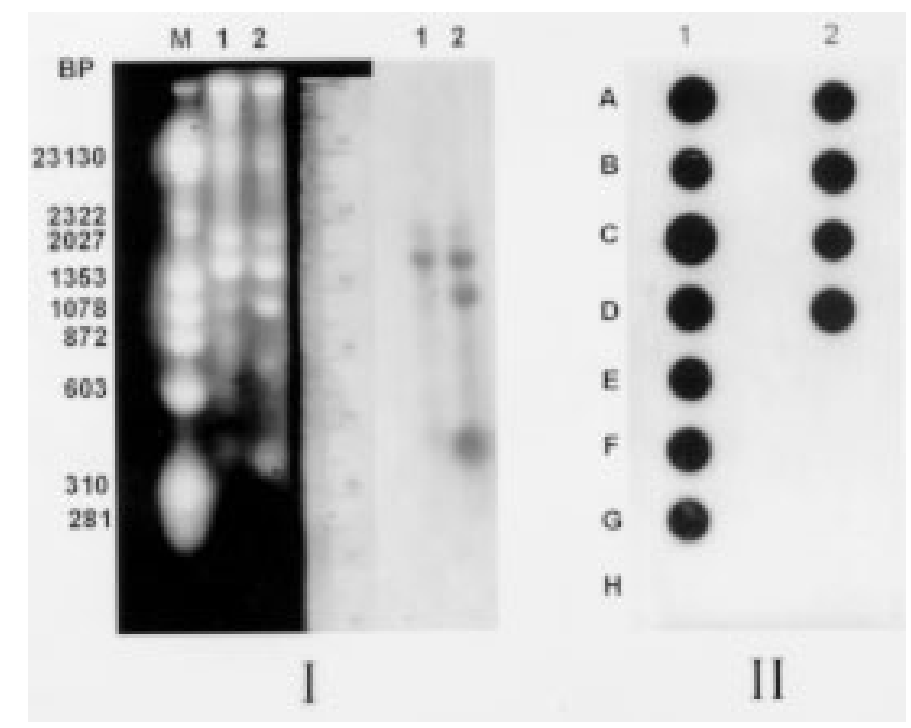

Fig. 3: Southern (I) and dot blot hybridization (II) after probing against total kDNA from CL strain and a specific synthetic oligonucleotide, respectively. I) Eco RI restriction fragments of kDNA and correspondent autoradiography: M: I Hind III / f x Hae III DNA marker; 1: parasite isolated from the patient; 2: CL strain. II) Different Trypanosoma (S.) cruzi isolates (20 ng of kDNA/dot) - 2D: parasite isolated from the patient; 1H: T. rangeli (negative control); 2E: Leishmania sp. (negative control).

\section{DISCUSSION}

In endemic areas the association of Chagas' disease and AIDS merits systematic study. The possibility of reactivation of dormant parasites with unusual clinical manifestations and dissimilar tropism (Amato et al. 1997) opens interesting fields for clinical-epidemiological studies as well as for investigations on biological behavior and genotypic variability of parasites isolated under immunosuppression situations. Particularly in Brazil, cases of HIV infections occur in large cities and the transmission of Chagas' disease is restricted to sylvatic and periurban areas. However, due to the expansion of HIV infection and the migration of individuals toward large urban centers, the occurence of a great number of AIDS cases with reactivation of this trypanosomiasis could be a real possibility.

The literature has reported the involvement of the central nervous system in cases of co-infection Chagas' disease/AIDS (Ferreira et al. 1991, Rocha et al. 1994) although cerebral lesions have only rarely been observed in chronic chagasic immunocompetent patients (Pittella 1985). Up to now, around 50 cases of CNS involvement in cases of association Chagas' disease/AIDS are known and have been reported at scientific meetings. From these, 13 have already been published (Rocha et al. 1994, Ferreira 1996). The first case of association between Chagas' disease/AIDS was published by Del Castillo et al. (1990) reporting the observation of a tumor in the cranial computed tomo- graphic scan of a patient. The first case considered as reactivation of Chagas' disease in a HIV positive patient proceeding from an endemic area was published by Gluckstein et al. (1992). The authors found a brain tumor from where T. cruzi was isolated.

Protozoan infections, against which immunity is basically T-cell mediated are likely to be more severe in individuals with AIDS than in immunocompetent hosts (Da-Cruz et al. 1992). Infection by $T$. cruzi usually reactivates when the CD4 T lymphocytes count is $£ 200$ cells $/ \mathrm{mm}^{3}$, similar to other opportunistic infections in AIDS. In the present study the patient presented decreasing levels of circulating CD4 cells, hence the possibility of reactivation of the disease is a plausible hypothesis. In addition, the finding of four cerebral lesions in our patient was similar to the observation reported by Gluckstein et al. (1992). Unfortunately, isolation of the parasite from the cerebral lesions was not possible since brain specimens were not cultured. However, T. cruzi was isolated from the blood and biochemically characterized.

The patient under investigation came from an urban area in the State of Minas Gerais considered as endemic for Chagas' disease. The strain isolated has shown to be genotypically similar to the $\mathrm{CL}$ strain based on restriction fragment length polymorphisms of kDNA and hybridization analyses suggesting that it probably belongs to the zymodeme 2 (Miles et al. 1980) which is found among strains that circulate in the domestic cycle 
of transmission. On the other hand, the Colombian strain is associated with the sylvatic cycle (zymodeme 1) and this fact may also explain the absence of cross-hybridization with the human isolate of the present study. As the patient had no history of blood transfusion he could not have experienced transfusion-associated Chagas' disease thus, the hypothesis of reactivation of a prior infection is strengthened. The genotypic (Morel et al. 1986) and phenotypic (Tibayrenc \& Ayala 1988) variability of circulating strains of $T$. cruzi can make it difficult to interpret the different manifestations of the disease and also to make correlations between strains and clinical symptoms. However, two zymodemes genetically related as Z2b (Y strain) and $\mathrm{Z} 2$ have been shown to be involved in inflammatory lesions of the ganglionic cells of the autonomic nervous system (Souza et al. 1996). The possibility of mixed infection with distinct subpopulations of $T$. cruzi in natural human infections can not be discarded (Morel et al. 1986). In addition, the distinct tissue tropism of $T$. cruzi lineages in mice provides support to the hypothesis that the genetic characteristics of $T$. cruzi clones are relevant to the pathogenesis of Chagas' disease (Andrade et al. 1997). Therefore, it is not unreasonable to speculate that in our patient a different subpopulation could be found in other organs or tissues.

To our knowledge, this is the first article reporting the genotypic characterization of $T$. cruzi, at the subgenus and strain levels, from a Brazilian HIV positive patient presenting involvement of the CNS by using a polymorphic DNA marker and molecular hybridization with specific probes. Although Gluckstein et al. (1992) have isolated $T$. cruzi from brain specimens, characterization of the parasite was not performed. A reaction with monoclonal fluorescent antibodies was used for discriminating between $T$. cruzi and $T$. rangeli. In addition, the present work point out for the reactivation of the trypanosomiasis by the demonstration of trypomastigote forms in the patient's blood. It is also possible that the cerebral lesions are indeed related to reactivation instead of being present prior to the immunosuppression. According to Pittella (1993), symptomatic acute forms accompanied by chagasic encephalitis are grave with death in virtually all cases and reactivation of the CNS infection in the chronic form of Chagas' disease occurs only in immunosuppressed patients. The parasite kDNA restriction pattern detected can be used as a marker for further comparisons with other $T$. cruzi strains from immunosuppressed and immunocompetent patients from the same and other endemic areas (Pacheco et al. unpublished results). Recently, Jimenez et al. (1995) reported the presence of cer- tain phenotypic patterns of parasites isolated from HIV positive patients. The authors adressed the possibility that the immune system could select virulent from avirulent populations. Corroborating with this hypothesis we have obtained evidence of selection of a subpopulation by investigating the biological behavior of the present $T$. cruzi strain. A parasite's subpopulation with a different genotypic profile has emerged during the reactivation of the disease after immunosuppression of chronic chagasic mice (Brito et al. 1996). On the other hand, Perez-Ramirez et al. (1997) reported that reactivation of Chagas' disease in HIV positive patient was not correlated with a particular genotype.

This paper reinforces the idea that cerebral Chagas' disease can be considered as another potential opportunistic infection in AIDS (Ferreira 1996) resulting from the reactivation of a dormant T. cruzi infection acquired years earlier. At the same time, it is a way of alerting physicians and researchers for the risk of reactivation of latent infections raising questions for future investigations on which parameters can be used to monitorize the infection and/or disease reactivation in immunosuppressed patients and when and how the parasites reactivates. Screening HIV positive patients from endemic areas for antibodies to T. cruzi may identify those at risk for this clinical manifestation (Gluckstein et al. 1992).

\section{ACKNOWLEDGEMENTS}

To Drs Douglas MacIntosh and Hooman Momen for the critical review of the manuscript.

\section{REFERENCES}

Aebischer T 1994. Recurrent cutaneous leishmaniasis: a role for persistent parasites? Parasitol Today 10: 25-28.

Alvar J 1994. Leishmaniasis and AIDS co-infection: the Spanish example. Parasitol Today 10: 160-163.

Amato JCP, Amato-Neto V, Amato VS, Duarte MIS, Uip DE, Boulos M 1997. Lesões cutâneas como únicas manifestações de reativação da infecção pelo Trypanosoma cruzi em receptora de rim por transplante. Rev Soc Bras Med Trop 30: 61-63.

Andrade LO, Chiari E, Machado CRS, Pena SDJ, Macedo AM 1997. Study of tissue tropism of Trypanosoma cruzi: Populations and its influence in the pathogenesis of Chagas' disease. Mem Inst Oswaldo Cruz 92 (Suppl. I): 258.

Brito CMM, Pires MQ, Da-Cruz AM, Pacheco RS 1996. Evidence of selection of subpopulations in Trypanosoma cruzi strains isolated from HIV positive patients. Mem Inst Oswaldo Cruz 91 (Suppl. I): 278.

Da-Cruz AM, Machado ES, Menezes JA, Rutowitsch MS, Coutinho SG 1992. Cellular and humoral immune reponses of a patient with American cutaneous leishmaniasis and AIDS. Trans $R$ Soc Trop Med Hyg 86: 511-512. 
Del Castillo M, Mendoza G, Oviedo J, Branco RP, Anselmo AE, Silva M 1990. AIDS and Chagas' disease with central nervous system tumor-like lesion. Am J Med 88: 693-694.

Dias JCP 1989. Control of Chagas' disease in Brazil. Parasitol Today 3: 336-341.

Ferreira MS 1996. The acquired immunodeficiency syndrome and endemic diseases in Brazil. Rev Soc Bras Med Trop 29: 531-535.

Ferreira MS, Nishioka AS, Rocha A, Silva AM, Ferreira RG, Olivier W, Tostes S 1991. Acute fatal Trypanosoma cruzi meningoencephalitis in a human immunodeficiency virus positive hemophiliac patient. $\mathrm{Am}$ J Trop Med Hyg 45: 723-727.

Gluckstein D, Ciferri F, Ruskin J 1992. Chagas' disease another cause of cerebral mass in the acquired immunodeficiency syndrome. Am J Med 92: 429-432.

Guevara P, Ramirez JL, Rojas E, Scorza JV, Gonzalez $\mathrm{N}$, Anez N 1993. Leishmania braziliensis in blood 30 years after cure. Lancet 341: 1341.

Jimenez M, Ferrer-Dufol M, Canavate C, Gutierrez-Solar B, Molina R, Laguna F, Lopez-Velez R, Cercenado E, Dauden E, Blasquez J, Guevara CL, Gomez J, Torre J, Barros C, Altes J, Serra T, Alvar J 1995. Variability of Leishmania (Leishmania) infantum among stocks from immunocompromised, immunocompetent patients and dogs in Spain. FEMS Microbiol Letters 131: 197-204.

Miles MA, Lanham SM, Souza AA, Povoa M 1980. Further enzymic characters of Trypanosoma cruzi and their evaluation for strain identification. Trans $R$ Soc Trop Med Hyg 74: 221-237.

Morel CM, Chiari E, Camargo EP, Mattei DM, Romanha AJ, Simpson L 1980. Strains and clones of Trypanosoma cruzi can be characterized by patterns of restriction endonuclease products of kinetoplast DNA minicircles. Proc Natl Acad Sci USA 77: 68106814.

Morel CM, Deane MP, Gonçalves AM 1986. The complexity of Trypanosoma cruzi populations revealed by schizodeme analysis. Parasitol Today 2: 97-101.

Pacheco RS, Lopes UG, Morel CM, Grimaldi Jr G, Momen H 1986. Schizodeme analysis of Leishmania isolates and comparison with some phenotypic techniques, p. 57-65. In JA Rioux, Leishmania Taxonomy et Phylogenese, Applications Eco-Epidemiologiques, IMEEE, Montpellier.

Pacheco RS, Brandão AA, Sibajev A, Cupolillo E, Momen H, Degrave W 1994. The genus Crithidia: genotypic diversity among species. J Protozool Res 4: 71-82.

Pacheco RS, Thomaz N, Brandão AA, Pires MQ, Momen H, Degrave W 1996. A synthetic oligonucleotide probe that discriminates between the subgenera Schizotrypanum and Megatrypanum. Parasite 3: 297-299.

PAHO-Pan American Health Organization 1990. Working group on AIDS case definition. Epidemiol Bull PAHO 10: 9.

Perez-Ramirez L, Barnabe C, Tibayrenc M 1997. The impact of HIV infection on Trypanosoma cruzi population structure. Mem Inst Oswaldo Cruz 92 (Suppl. I): 276.

Pittella JEH 1985. Brain involvement in the chronic cardiac form of Chagas' disease. J Trop Med Hyg 88: 313-317.

Pittella JEH 1993. The central nervous system involvement in Chagas' disease: an updatting. Rev Inst Med Trop São Paulo 35: 111-116.

Rocha A, Meneses ACO, Silva AM, Ferreira MS, Nishioka AS, Burgarelli MKN, Almeida E, Turcato Jr G, Metze K, Lopes ER 1994. Pathology of patients with Chagas' disease and acquired immunodeficiency syndrome. Am J Trop Med Hyg 50: 261-268.

Saravia NG, Weigle K, Segura I, Giannini SH, Pacheco RS, Labrada LA, Gonçalves AM 1990. Recurrent lesions in human Leishmania braziliensis infections: reactivation or reinfection? Lancet 336: 398-402.

Souza MM, Andrade SG, Barbosa Jr AA, Santos RTM, Ferreira VA, Andrade ZA 1996. Influence of Trypanosoma cruzi strains on autonomic nervous system pathology (an experimental study in mice). Mem Inst Oswaldo Cruz 92: 217-224.

Tibayrenc M, Ayala FJ 1988. Isozyme variability in Trypanosoma cruzi the agent of Chagas' disease: genetical, taxonomical and epidemiological significance. Evolution 42: 277-279. 
Chagas' Disease and HIV Co-infection - Raquel S Pacheco et al. 